\title{
Electroencephalogram-derived pain index for evaluating pain during labor
}

\author{
Liang Sun ${ }^{1}$, Hong Zhang ${ }^{1}$, Qiaoyu Han ${ }^{1}$, Yi Feng ${ }^{\text {Corresp. } 1}$ \\ ${ }^{1}$ Department of Anesthesiology, Peking University People's Hospital, Beijing, China \\ Corresponding Author: Yi Feng \\ Email address: yifeng65@outlook.com
}

Background: The discriminative ability of a point-of-care electroencephalography (EEG)derived pain index (Pi) for objectively assessing pain has been validated in chronic pain patients. The current study aimed to determine its feasibility in assessing labor pain in an obstetric setting. Methods: Parturients were enrolled from the delivery room at the department of obstetrics in a tertiary hospital between February and June of 2018. Pi values and relevant numerical rating scale (NRS) scores were collected at different stages of labor in the presence or absence of epidural analgesia. The correlation between $\mathrm{Pi}$ values and NRS scores was analyzed using the Pearson correlation analysis. The receiver operating characteristic (ROC) curve was plotted to estimate the discriminative capability of Pi to detect labor pain in parturients. Results: Eighty paturients were eligible for inclusion. The Pearson correlation analysis exhibited a positive correlation between $\mathrm{Pi}$ values and NRS scores in parturients $(r=0.768, P<0.001)$. The ROC analysis revealed a cut-off Pi value of 18.37 to discriminate between mild and moderate-to-severe labor pain in parturients. Further analysis indicated that Pi values had the best diagnostic accuracy reflected by the highest area under the curve (AUC) of 0.857 , with a sensitivity and specificity of 0.767 and 0.833 , respectively, and a Youden index of 0.6. Subgroup analyses further substantiated the correlations between Pi values and NRS scores, especially in parturients with higher pain intensity. Conclusion: This study indicates that Pi values derived from EEGs significantly correlate with the NRS scores, and can serve as a way to quantitatively and objectively evaluate labor pain in parturients. 
1 Electroencephalogram-derived pain index for evaluating pain during labor

2 Liang Sun; Hong Zhang; Qiaoyu Han; Yi Feng

3 Department of Anesthesiology, Peking University People's Hospital, Beijing 100044,

4 China

5 Corresponding author:

$6 \quad$ Yi Feng

7 No.11, Xi Zhi Men Nan Da Jie, Xicheng District, Beijing 100044, China.

8 Email address: yifeng65@outlook.com

9 Abstract

Background: The discriminative ability of a point-of-care electroencephalography (EEG)derived pain index $(\mathrm{Pi})$ for objectively assessing pain has been validated in chronic pain patients. The current study aimed to determine its feasibility in assessing labor pain in an obstetric setting.

Methods: Parturients were enrolled from the delivery room at the department of obstetrics in a tertiary hospital between February and June of 2018. Pi values and relevant numerical rating scale (NRS) scores were collected at different stages of labor in the presence or absence of epidural analgesia. The correlation between Pi values and NRS scores was analyzed using the Pearson correlation analysis. The receiver operating characteristic (ROC) curve was plotted to estimate the discriminative capability of $\mathrm{Pi}$ to detect labor pain in parturients.

Results: Eighty paturients were eligible for inclusion. The Pearson correlation analysis exhibited a positive correlation between Pi values and NRS scores in parturients ( $r=$ $0.768, \mathrm{P}<0.001)$. The ROC analysis revealed a cut-off $\mathrm{Pi}$ value of 18.37 to discriminate 
24

between mild and moderate-to-severe labor pain in parturients. Further analysis indicated that Pi values had the best diagnostic accuracy reflected by the highest area under the curve (AUC) of 0.857 , with a sensitivity and specificity of 0.767 and 0.833 , respectively, and a Youden index of 0.6. Subgroup analyses further substantiated the correlations between Pi values and NRS scores, especially in parturients with higher pain intensity.

Conclusion: This study indicates that Pi values derived from EEGs significantly correlate with the NRS scores, and can serve as a way to quantitatively and objectively evaluate labor pain in parturients.

\section{Introduction}

The pain experienced during labor has been described as one of the most painful events in a woman's life, and can be excruciating (Chan et al., 2019). The literature characterizes a vast array of factors which affect the perception of labor pain, including psychological mechanisms, duration of labor, fetus weight, and gene polymorphisms (George et al., 2013; Terkawi et al., 2014). Labor pain can also adversely impact the childbirth experience. In a severe pain scenario,_labor pain can engender psychological stress, and in some cases, negatively interfere with the normal process of labor, thereby increasing the rate of cesarean section (Labor \& Maguire, 2008).

Timely and precise pain assessment plays a pivotal role in well-orchestrated pain management. Finding optimal ways to alleviate pain and thus improve both the physical and psychological well-being of people with pain is crucial to pain management (Erden et al., 2017).

To date, there are no widely accepted methods of objectively assessing pain, so a 
47 battery of subjective assessments such as the visual analog scale (VAS), the verbal 48 rating scale (VRS), and the numerical rating scale (NRS) are generally used (Kaibori et

al., 2015). These subjective scales, however, are affected by subjective influences, evaluation lag, and many other factors. Accordingly, a wide range of objective physiological indicators including heart rate variability, blood pressure variability, nociception level (NoL) index, analgesia nociception index (ANI), skin electrical conductivity, and other possible indicators for pain monitoring have been developed (Stockle et al., 2018; Edry et al., 2016; An et al., 2017). However, these pain-monitoring indicators are also affected by a broad spectrum of factors and thus generate disappointing results when trying to accurately assess pain. Therefore, a more reliable objective, quantitative indicator is urgently need.

Several noninvasive imaging devices merit consideration (e.g., positron emission tomography, functional magnetic resonance imaging), however, as argued in the literature, there is still disagreement about the degree to which current measures of brain activity exactly relate to pain. Given the high temporal resolution of electroencephalograms (EEGs) and their ability to capture real-time changes within the brain, they are regarded as the most promising neuroimaging devices for an objective pain diagnosis (Benoit et al., 2017; Okolo \& Omurtag, 2018; Reches et al., 2016; Levitt \& Saab, 2019).

The Pain index (Pi, Beijing Easymonitor Technology Co., Ltd, Beijing, China), a novel point-of-care indicator, was developed from the whole frequency band-EEG wavelet algorithm. A previous study confirmed that Pi significantly correlated with NRS scores in chronic pain patients, and thus provided valuable insights into the possibility of objective 
70 pain assessment in clinical settings (An et al., 2017). Labor pain differs significantly

71 from chronic pain. According to its definition, labor pain consists of two discomfort

72 dimensions: a sense of physical pain intensity and a psychological stress state.

73 Moreover, parturients undergo different trajectories and mechanisms of pain during the

74 first and second stages of labor (Kafshdooz et al., 2019). Specifically, the pain during

75 the first stage of labor is largely visceral in origin and cannot be localized well, whereas

76 during the transitional and second stages, the somatic pain becomes more intense and

77 well located in the lower part of the abdomen (Yuksel et al., 2017). The reliability and

78 validity of $\mathrm{Pi}$ for assessing labor pain in the obstetric setting require further clarity and

because of the nature of labor pain, parturients are a natural model for a study of Pi that

80 includes both visceral and somatic pain. Building on these considerations, we extended

81 our line of investigation to identify the correlation between Pi values and NRS scores in

82 parturients, as well as explore the discriminative ability of $\mathrm{Pi}$ to detect both visceral and

83 somatic pain.

84 Methods

85 This study was approved by the Institution Review Board (Ethics Committee of Peking University People's Hospital, Peking University, No. 2016PHB031-01) and registered on

87 the Chinese Clinical Trials Registry (ChiCTR1800014769). Informed written consent was obtained from all participants. The flow chart of study participation was shown in

89 Fig. 1.

90 Study procedure

91 Parturients who were admitted to the delivery room at the Department of Obstetrics,

92 Peking University People's Hospital between February and June of 2018 were 
93 consecutively enrolled, and epidural analgesia was implemented according to the

94 requirements of the parturients.

95 Inclusion criteria were: (1) women who were 21-39 years old; (2) had a gestational age

96 of 37-42 weeks; (3) were prepared to deliver vaginally with a single, cephalic, term

97 pregnancy; (4) parturients who entered the first or second stage of labor; (5) and were

98 admitted to the delivery room during daytime working hours (between 8 am to $5 \mathrm{pm}$ ).

99 Exclusion criteria included: (1) a history of psychiatric disease (those who were

100 diagnosed before or during pregnancy by a psychiatrist); (2) or those Incapable of giving

101 consent.

102 Demographic and clinical data were collected including: maternal age, height, weight,

103 gestational age, parity, stages of labor, and epidural analgesia information. Since pain

104 complaints are usually reported by the parturient during the first and second stage of

105 labor, and since the third stage lasts for a very short time (usually less than $30 \mathrm{~min}$ ) and

106 is usually accompanied with mild pain, we did not collect the relevant data during the

107 third stage. In addition to blood pressure and oxygen saturation as measured by pulse

108 oximetry $\left(\mathrm{SpO}_{2}\right)$, real-time $\mathrm{Pi}$ values were collected from a multifunction monitor (Beijing

109 Easymonitor Technology Co., Ltd, Beijing, China) with acquisition electrodes.

110 Parturients were asked to quantify their pain intensity using a 0-to-10-point NRS (with

111 "0" representing no pain and "10" the worst pain; an NRS score $\leq 3$ corresponds to mild

112 pain, 4-6 to moderate pain, and 7-10 to severe pain (Boonstra et al., 2016) ) scale at

113 different time points (initiation, apex and interval of three consecutive uterine

114 contractions) during either the first or second stage of labor aided by a tocometer. If the

115 parturient refused to describe their pain due to severe pain, the NRS score was 
116 permitted by a subsequent recall when the pain subsided. The Pi values (0-100) at THE

117 above-mentioned time points were also recorded.

118 EEG collection and Pi calculation

119 EEG signals were simultaneously monitored and collected by 5 electrodes, according to

120 the manufacturer instructions and a previous study (Wang et al., 2020). There were 3

121 electrodes on each subject's forehead: one located $2 \mathrm{~cm}$ above the midpoint, and two

122 placed above the bilateral eyebrows. Meanwhile, two reference electrodes were placed

123 on the bilateral mastoid processes (Fig. 2a, b). The raw EEG signals were collected

124 and further processed for the calculation of Pi values.

125 The EEG analysis software package (Beijing Easymonitor Technology Co., Ltd., Beijing,

126 China) was applied, based on the whole frequency band EEG wavelet algorithm, which

127 is currently one of the most suitable tools for analyzing EEGs (Zhang et al., 2010;

128 Constant \& Sabourdin, 2012). The algorithm formulas and calculation methods of raw

129 EEG data processing were discussed previously (An et al., 2017), and the details can

130 be found in Supplemental Information 2. Pi values were displayed on the monitor

131 screen in a real-time manner (Fig. 2c), with values updating every 2 seconds (sampling

132 rate: $1600 \mathrm{~Hz}$ ). Moreover, taking into account that EEGs are susceptible to the impact of

133 the electromyography (EMG), EMG components were filtered during Pi value

134 calculation.

135 Statistical analysis

136 A sample size of $n=70$ parturients was calculated according to a minimal detectable

137 correlation between Pi values and NRS scores of $r=0.4$, with an $\alpha$ error of $5 \%$ and a 
138 power of $80 \%$. In total, we included 84 participants to allow for an approximate $20 \%$ loss

139 due to protocol violation, parturient withdrawal, or technique-related problems.

140 Data are presented as the mean \pm standard deviations (SDs) for normally distributed

141 continuous variables or median [interquartile range] if distributions were skewed, while

142 categorical variables are expressed as frequencies or percentages. Normal distribution

143 of the data was tested using the Kolmogorov-Smirnov test. The degree of dependency

144 between variables was estimated using the Pearson correlation test. In addition, the

145 receiver operating characteristic (ROC) curve was applied to test Pi's ability to

146 discriminate between subjects who will and those who would develop moderate or

147 severe labor pain (NRS>3) and those who would not. The area under the curve (AUC)

148 estimates were also determined in order to indicate the probability of accurately

149 discriminating between the different pain groups. The Youden index was calculated and

150 defined as the value of "sensitivity + specificity-1". In general, the optimal cut-off value

151 was calculated according to the ROC curve analysis, maximizing the Youden index

152 (Youden, 1950). The SPSS 19.0 (SPSS Inc., Chicago, IL, USA) package was used, and

153 a $\mathrm{P}<0.05$ was considered statistically significant.

\section{Results}

\section{Clinical characteristics of parturients}

156 A total of 80 parturients fulfilled the inclusion criteria and were enrolled into the final

157 analysis; 4 patients were excluded because of either an incapacity to consent $(n=3)$ or a 158 technical problem $(n=1)$. Detailed demographic and clinical characteristics of all

159 parturients were shown in Table 1. Finally, the NRS scores and Pi values at the same 160 time points were averaged for further analysis. 
161 Correlation of $\mathrm{Pi}$ value with NRS score in parturients

162 The Pearson correlation test showed a positive correlation between Pi value and NRS

163 score in the enrolled parturients $(r=0.768, P<0.001$, Fig. 3$)$, suggesting that $P i$ could

164 be used for pain monitoring in parturients.

165 Subgroup analyses for correlation of Pi values with NRS score controlling for 166 different confounding factors

167 In order to further substantiate the correlation of Pi values with NRS scores, subgroup 168 analyses were performed taking the underlying confounding factors into account.

169 a. Different stages of labor

170 During the different stages of labor, the correlation between Pi values and NRS score 171 were as follows: $a$. the first stage of labor $(r=0.741, P<0.001)$; $b$. the second stage of 172 labor $(r=0.774, P<0.001)$ (Fig. 4a and 4b).

173 b. The presence or absence of analgesia

174 The Pearson correlation test that considered the possible impact of epidural analgesia 175 also exhibited a positive correlation between Pi value and NRS score: $\mathrm{c}$. the presence 176 of analgesia $(r=0.760, P<0.001)$; $d$. the absence of analgesia $(r=0.750, P<0.001)$, as

177 shown in Fig. 4c and 4d.

178 c. Different time points of uterine contractions

179 As for uterine contractions, the correlation between Pi value and NRS score varied

180 between the different time points as follows: e. the initiation of uterine contraction

$181(r=0.582, P<0.001)$; $f$. the apex of uterine contraction $(r=0.751, P<0.001)$; $g$. the interval

182 of uterine contraction ( $r=0.487, P<0.001)$, as shown in Fig. $\mathbf{4 e}, \mathbf{4 f}$ and $\mathbf{4 g}$. These results 
183 suggest that the correlation between Pi values and NRS score was stronger at the apex

184 of uterine contractions compared to the initiation and interval of uterine contraction.

185

186

187

188

189

190

191

192

193

194

195

196

197

198

199

200

201

202

203

204

205

\section{d. Pain intensity}

As discussed previously, an NRS score < 4 corresponds to mild pain and an NRS

score $\geq 4$ indicates a moderate or severe pain. With respect to pain intensity, the

Pearson correlation test showed a stronger correlation between Pi value and NRS score in parturients with moderate or severe pain compared to those with mild pain: $h$. NRS score $<4(r=0.386, P<0.001)$; i. NRS score $\geq 4$. $(r=0.688, P<0.001)$, as shown in Fig. 4h and $4 \mathbf{i}$.

\section{Ability of Pi to discriminate between mild and moderate-to-severe labor pain}

According to the ROC analysis, we evaluated the capability of $\mathrm{Pi}$ to distinguish between mild and moderate-to-severe labor pain in parturients. The ROC curve constructed using Pi revealed that the AUC was 0.857 (Fig. 5a), and the optimal cut-off value of Pi was 18.37, with a sensitivity, specificity and Youden index of $0.767,0.833$ and 0.6 , respectively. Moreover, when the cut-off value was set to 15.0 , the sensitivity was the highest (0.825). There was, however, a lower specificity and Youden index of 0.633 and 0.458, respectively (Table 2 ).

Subgroup analyses of ability of $\mathrm{Pi}$ to discriminate between mild and moderate-tosevere labor pain in different scenarios

We also performed subgroup analyses to strengthen the diagnostic ability of Pi to discriminate between mild and moderate-to-severe labor pain in parturients.

\section{a. Different stages of labor}


206 The ROC analysis also demonstrated that Pi could distinguish between mild and

207 moderate-to-severe labor pain in parturients at different stages of labor: $b$. the first stage

208 of labor $(A \cup C=0.819,95 \% \mathrm{Cl}: 0.756-0.882)$; c. the second stage of labor $(A \cup C=0.87,95 \%$

209 Cl: 0.764-0.975), as shown in Fig. 5b and 5c.

210 b. The presence or absence of analgesia

211 Taking into account the impact of analgesia, the ROC analysis showed that Pi could

212 distinguish between mild and moderate-to-severe labor pain in parturients with or

213 without epidural analgesia: $d$. the presence of analgesia $(A \cup C=0.852,95 \% \mathrm{Cl}: 0.746-$

214 0.958); e. the absence of analgesia ( $\mathrm{AUC}=0.849,95 \% \mathrm{Cl}: 0.793-0.904)$, indicating that

215 there was no influence of analgesia on the diagnostic performance of Pi (Fig. $5 \mathbf{d}$ and 216 5e).

\section{7 c. Different time points of uterine contractions}

218 The ROC analysis considered the time points of uterine contraction and revealed that $\mathrm{Pi}$

219 could distinguish between mild and moderate-to-severe labor pain in parturients at

220 different time points during uterine contractions. The highest diagnostic value was at the

221 apex of contraction: $f$. the initiation of uterine contraction $(A \cup C=0.816,95 \% \mathrm{Cl}: 0.721-$

222 0.911); g. the apex of uterine contraction (AUC=0.855, 95\% Cl: $0.770-0.939)$; h. the

223 interval of uterine contraction $(\mathrm{AUC}=0.770,95 \% \mathrm{Cl}: 0.634-0.906)$ as shown in Fig. $\mathbf{5 f}$,

$2245 \mathrm{~g}$ and $5 \mathrm{~h}$.

225 Discussion

226 Our results from the current study support the notion that Pi values, extracted from

227 EEGs, exhibited a positive correlation with NRS scores in parturients. Following a

228 previous study conducted in a chronic pain setting (An et al., 2017), the diagnostic 
229 accuracy of Pi was powerful enough to apply the cut-off value clinically (the sensitivity,

230 specificity and Youden index were $0.767,0.833$ and 0.6 , respectively).

231 Although complex modulation is implicated in the pain conduction pathways, in theory,

232 EEGs can collect information on neuronal activation, evaluate the components of pain

233 activity, and thus provide a feasible method for processing complex pain information

234 (Wagemakers et al., 2019; Levitt \& Saab, 2019). However, the bispectral index (BIS), an

235 EEG-based variable widely used in the general anesthesia setting, can only provide

236 invaluable inference for the level of consciousness of the patients and so fails to serve

237 as an accurate indicator of nociceptive stimulation. Currently, there is no advanced,

238 reliable, objective and quantitative way to measure pain in clinical practice (An et al.,

239 2017). In this study, we collected EEG signals from the prefrontal lobe using two

240 channels (left and right) and further extracted the pain components, exhibiting the

241 reliability and validity of $\mathrm{Pi}$ for diagnosing labor pain.

242 Evidence suggests that pain can cause significant and specific changes to multi-brain

243 regions and multi-frequency EEG signals (Roberts et al., 2008; Mouraux \& lannetti,

244 2018). Li et al conducted continuous EEG recordings on 19 healthy subjects and found

245 a strong correlation between the placebo effect on reported pain perception and alpha

246 amplitude, which suggested that alpha oscillations in frontal-central regions serve as a

247 cortical oscillatory basis of the placebo effect on tonic muscle pain (Li et al., 2016).

248 Likewise, significant overactivation of the pain network was detected in multiple brain

249 regions [e.g., parietal lobule, anterior cingulate, thalamus, anterior and posterior insula,

250 dorsolateral prefrontal cortex (DLPFC) and S1] in a large population of chronic

251 neuropathic pain patients using quantitative EEG, which was in line with conventional 
252 functional neuroimaging findings and extended to cover the mid and posterior cingulate,

253 supporting the notion that the enhanced temporal resolution of electrophysiological

254 methods may facilitate more precise identification and evaluation of the pain network

255 (Prichep et al., 2018). Similarly, correlations of pain perception and functional alterations

256 in the brain (mainly identified using EEG) have also been implicated in other pain

257 settings, including visceral pain and fibromyalgia (Mayer et al., 2015). A recent study

258 investigating a novel pain recognition indicator, the pain threshold index (PTI), which is

259 based on the same principle as $\mathrm{Pi}$, revealed a better predictive accuracy for

260 postoperative pain than THE surgical pleth index (SPI) and could differentiate

261 moderate-to-severe pain from mild pain in patients who had recovered from general

262 anesthesia. Therefore, together with our findings, the current evidence lends increasing

263 support for the potential role of EEG as an objective pain indicator, which includes

264 indicators extracted from EEG like Pi.

265 In addition, if available, epidural analgesia remains a highly requested modality for pain

266 relief during labor (Traynor et al., 2016), and performing pain evaluations during

267 epidural analgesia is a big concern. Therefore, we performed subgroup analyses taking

268 confounding factors into account (e.g., stages of labor, analgesia, uterine contractions

269 and pain intensity), and our results further substantiated a robust correlation between $\mathrm{Pi}$

270 values and NRS scores, positing that $\mathrm{Pi}$ is suitable for evaluating the labor pain of

271 parturients. Specifically, our study showed a stronger correlation and higher diagnostic

272 ability in parturients with a higher pain intensity (those with NRS $\geq 4$ or at the apex of

273 uterine contraction) than those with mild pain. Markedly, we calculated a cut-off value of

27418.37 for predicting labor pain under the optimal Youden index. However, considering 
275 the unique characteristics of labor pain, in combination with the principle of early

276 diagnosis and treatment, we strongly recommended the cut-off value of $\mathrm{Pi}$ be set at 15.0,

277 with the highest sensitivity (0.825), even with a lower specificity.

278 Our study has several limitations. First, our study population was relatively small, and

279 more reliable results could likely be obtained with more participants. Second, we

280 omitted some potential confounding factors from our study (e.g., fetus weight, the third

281 stage of labor, etc.), possibly causing e risk bias, which merits future investigation. Third,

282 we employed a tocometer to judge the time points of initiation, termination, and apex of

283 each uterine contraction. We believe that the tocometer is currently the most accurate

284 method for judging these time points, but some may disagree. For future research, a

285 well-designed, large-scale, multi-center study is still needed to further validate the

286 feasibility of $\mathrm{Pi}$ in an obstetric setting.

287 Conclusion

288 In summary, the current study confirmed that $\mathrm{Pi}$, based on the EEG wavelet algorithm, 289 as a noninvasive, objective, point-of-care monitoring indicator can reflect the existence 290 and the severity of pain in parturients. Pi might serve as a potential alternative in the 291 future to help clinicians quantitatively and objectively assess labor pain.

\section{References}

293 An JX, Wang Y, Cope DK, Williams JP. 2017. Quantitative evaluation of pain with pain 294 index extracted from electroencephalogram. Chinese Medical Journal 130(16):1926-

2951931 DOI 10.4103/0366-6999.211878. 
296 Benoit B, Martin-Misener R, Newman A, Latimer M, Campbell-Yeo M. 2017.

297 Neurophysiological assessment of acute pain in infants: a scoping review of research

298 methods. Acta Paediatrica 106(7):1053-1066 DOI 10.1111/apa.13839.

299 Boonstra AM, Stewart RE, Köke AJ, Oosterwijk RF, Swaan JL, Schreurs KM,

300 Schiphorst Preuper HR. 2016. Cut-off points for mild, moderate, and severe pain on the

301 numeric rating scale for pain in patients with chronic musculoskeletal pain: variability

302 and influence of sex and catastrophizing. Frontiers in Psychology 7:1466 DOI

$303 \quad 10.3389 /$ fpsyg.2016.01466.

304 Chan J, Gan YY, Dabas R, Han NR, Sultana R, Sia A, Sng BL. 2019. Evaluation of

305 association factors for labor episodic pain during epidural analgesia. Journal of Pain

306 Research 12:679-687 DOI 10.2147/JPR.S185073.

307 Constant I, Sabourdin N. 2012. The EEG signal: a window on the cortical brain activity.

308 Paediatric Anaesthesia 22(6):539-552 DOI 10.1111/j.1460-9592.2012.03883.x.

309 Edry R, Recea V, Dikust Y, Sessler DI. 2016. Preliminary intraoperative validation of the

310 nociception level index: a noninvasive nociception monitor. Anesthesiology 125(1):193-

311203 DOI 10.1097/ALN.0000000000001130.

312 Erden S, Demir SG, Kanatlı U, Danacı F, Carboğa B. 2017. The effect of standard pain

313 assessment on pain and analgesic consumption amount in patients undergoing

314 arthroscopic shoulder surgery. Applied Nursing Research 33:121-126 DOI

315 10.1016/j.apnr.2016.11.009.

316 George RB, Allen TK, Habib AS. 2013. Intermittent epidural bolus compared with

317 continuous epidural infusions for labor analgesia: a systematic review and meta- 
318 analysis. Anesthesia and Analgesia 116(1):133-144 DOI

319 10.1213/ANE.0b013e3182713b26.

320 Kafshdooz L, Kahroba H, Kafshdooz T, Sheervalilou R, Pourfathi H. 2019. Labour

321 analgesia; Molecular pathway and the role of nanocarriers: a systematic review.

322 Artificial cells, Nanomedicine, and Biotechnology 47(1):927-932 DOI

$32310.1080 / 21691401.2019 .1573736$.

324 Kaibori M, lida H, Matsui K, Ishizaki M, Matsushima H, Sakaguchi T, Fukui J, Inoue K,

325 Matsui $Y$, Kon M. 2015. [Objective assessment of postoperative pain after digestive

326 tract surgery]. Nihon Geka Gakkai Zasshi 116(4):243-248.

327 Labor S, Maguire S. 2008. The pain of labour. Reviews in Pain 2(2):15-19 DOI

328 10.1177/204946370800200205.

329 Levitt J, Saab CY. 2019. What does a pain 'biomarker' mean and can a machine be 330 taught to measure pain. Neuroscience Letters 702:40-43 DOI

331 10.1016/j.neulet.2018.11.038.

332 Li L, Wang H, Ke X, Liu X, Yuan Y, Zhang D, Xiong D, Qiu Y. 2016. Placebo analgesia 333 changes alpha oscillations induced by tonic muscle pain: EEG frequency analysis 334 including data during pain evaluation. Frontiers in Computational Neuroscience 10:45 335 DOI 10.3389/fncom.2016.00045.

336 Mayer EA, Gupta A, Kilpatrick LA, Hong JY. 2015. Imaging brain mechanisms in chronic 337 visceral pain. Pain 156 Suppl 1(0 1):S50-S63 DOI 10.1097/j.pain.0000000000000106.

338 Mouraux A, lannetti GD. 2018. The search for pain biomarkers in the human brain. 339 Brain 141(12):3290-3307 DOI 10.1093/brain/awy281. 
340 Okolo C, Omurtag A. 2018. Research: use of dry electroencephalogram and support

341 vector for objective pain assessment. Biomedical Instrumentation \& Technology

$342 \quad 52(5): 372-378$ DOI 10.2345/0899-8205-52.5.372.

343 Prichep LS, Shah J, Merkin H, Hiesiger EM. 2018. Exploration of the pathophysiology of 344 chronic pain using quantitative EEG source localization. Clinical EEG and Neuroscience 345 49(2):103-113 DOI 10.1177/1550059417736444.

346 Reches A, Nir RR, Shram MJ, Dickman D, Laufer I, Shani-Hershkovich R, Stern Y,

347 Weiss M, Yarnitsky D, Geva AB. 2016. A novel electroencephalography-based tool for 348 objective assessment of network dynamics activated by nociceptive stimuli. European 349 Journal of Pain 20(2):250-262 DOI 10.1002/ejp.716.

350 Roberts K, Papadaki A, Gonçalves C, Tighe M, Atherton D, Shenoy R, McRobbie D, 351 Anand P. 2008. Contact heat evoked potentials using simultaneous Eeg and Fmri and 352 their correlation with evoked pain. BMC Anesthesiology 8:8 DOI 10.1186/1471-2253-8-8.

353 Stöckle PA, Julien M, Issa R, Décary E, Brulotte V, Drolet P, Henri M, Poirier M, 354 Latulippe JF, Dorais M, Verdonck O, Fortier LP, Richebé P. 2018. Validation of the 355 PMD100 and its NOL index to detect nociception at different infusion regimen of 356 remifentanil in patients under general anesthesia. Minerva Anestesiologica 84(10):11603571168 DOI 10.23736/S0375-9393.18.12720-9.

358 Terkawi AS, Jackson WM, Hansoti S, Tabassum R, Flood P. 2014. Polymorphism in the 359 ADRB2 gene explains a small portion of intersubject variability in pain relative to 360 cervical dilation in the first stage of labor. Anesthesiology 121(1):140-148 DOI 361 10.1097/ALN.0000000000000258. 
362 Traynor AJ, Aragon M, Ghosh D, Choi RS, Dingmann C, Vu Tran Z, Bucklin BA. 2016.

363 Obstetric Anesthesia Workforce Survey: a 30-year update. Anesthesia and Analgesia

364 122(6):1939-1946 DOI 10.1213/ANE.0000000000001204.

365 Wagemakers SH, van der Velden JM, Gerlich AS, Hindriks-Keegstra AW, van Dijk J,

366 Verhoeff J. 2019. A systematic review of devices and techniques that objectively

367 measure patients' pain. Pain Physician 22(1):1-13.

368 Wang R, Deng Y, Zhou S, Zhang J. 2020. EEG-derived pain threshold index for

369 prediction of postoperative pain in patients undergoing laparoscopic urological surgery:

370 a comparison with surgical pleth index. Journal of Clinical Monitoring and Computing

371 DOI 10.1007/s10877-020-00604-w.

372 Youden WJ. 1950. Index for rating diagnostic tests. Cancer 3(1):32-35 DOI

373 10.1002/1097-0142(1950)3:1\&lt;32::aid-cncr2820030106\&gt;3.0.co;2-3.

374 Yuksel H, Cayir Y, Kosan Z, Tastan K. 2017. Effectiveness of breathing exercises

375 during the second stage of labor on labor pain and duration: a randomized controlled

376 trial. Journal of Integrative Medicine 15(6):456-461 DOI 10.1016/S2095-4964(17)60368-

3776.

378 Zhang XT, Cheng H, Xiong W, Wang BG. 2010. Comparison of the ability of wavelet

379 index and bispectral index for reflecting regain of consciousness in patients undergone 380 surgery. Chinese Medical Journal 123(12):1520-1523. 


\section{Table $\mathbf{1}$ (on next page)}

Table 1 The clinical characteristics of included parturients $(n=80)$.

Notes. Data are expressed as mean \pm standard deviations (SDs) or frequencies (percentages). 


\begin{tabular}{ll}
\hline Variables & Values \\
\hline Age (years) & $32.1 \pm 4.0$ \\
Height $(\mathrm{cm})$ & $162.3 \pm 4.8$ \\
Weight $(\mathrm{kg})$ & $73.0 \pm 12.1$ \\
Gestational age (day) & $274.7 \pm 15.8$ \\
Primipara $\mathrm{n}(\%)$ & $70(87.5 \%)$ \\
Epidural analgesia $\mathrm{n}(\%)$ & $21(26.3 \%)$ \\
First stage of labor $\mathrm{n}(\%)$ & $60(75 \%)$ \\
Second stage of labor $\mathrm{n}(\%)$ & $20(25 \%)$ \\
\hline
\end{tabular}




\section{Table 2 (on next page)}

The capability of different Pi cutoff values to distinguish labor pain in parturients.

Notes. The Youden index was calculated and defined as the value of "sensitivity + specificity-1". The optimal cut-off value was calculated, maximizing the Youden index. 


\begin{tabular}{lccc}
\hline Cutoff & Sensitivity & Specificity & Youden index \\
\hline 15.00 & 0.825 & 0.633 & 0.458 \\
16.08 & 0.783 & 0.700 & 0.483 \\
16.99 & 0.775 & 0.742 & 0.517 \\
18.37 & 0.767 & 0.833 & 0.600 \\
19.31 & 0.717 & 0.850 & 0.567 \\
20.11 & 0.708 & 0.867 & 0.575 \\
21.07 & 0.683 & 0.875 & 0.558 \\
22.01 & 0.650 & 0.917 & 0.567 \\
\hline
\end{tabular}




\section{Figure 1}

The flow diagram of the study protocol.

$\mathrm{Pi}=$ pain index, NRS= numerical rating scale.

Eligibility: parturients scheduled for a spontaneous labor $(n=84)$

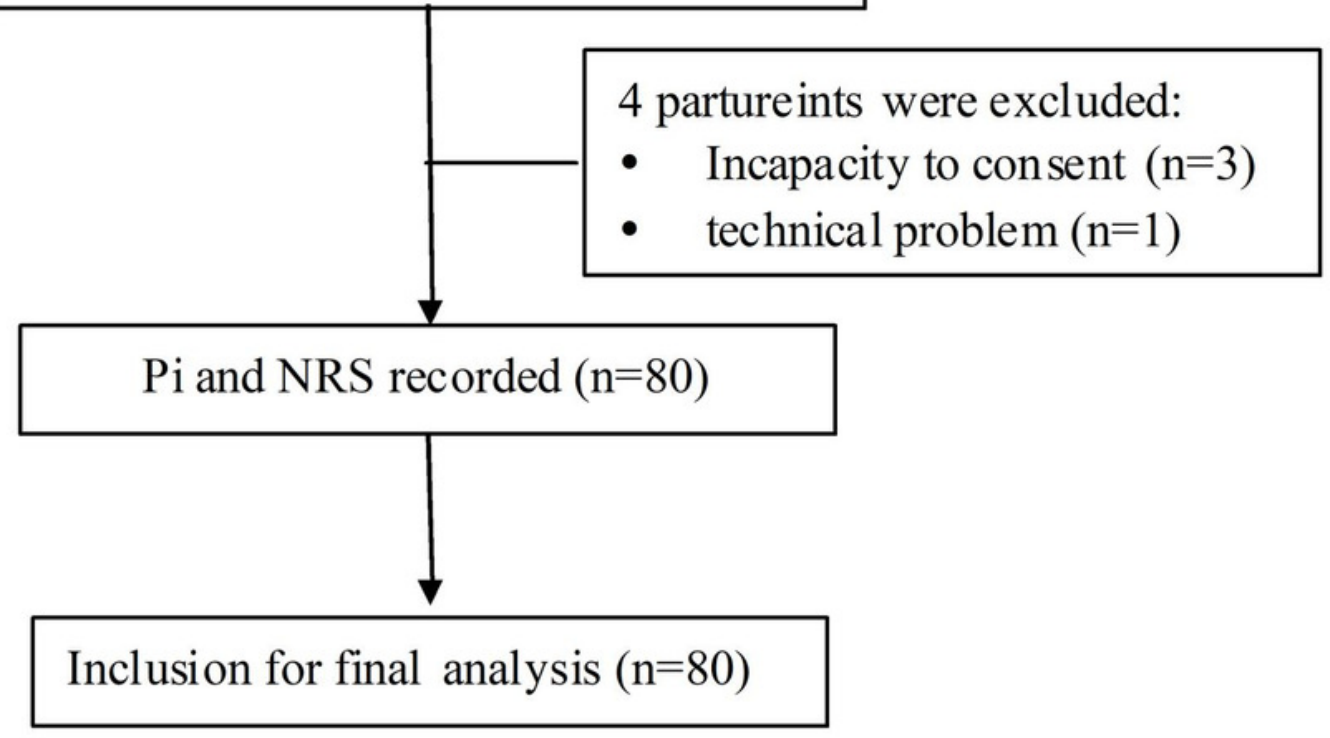




\section{Figure 2}

Placement of EEG electrodes and display of Pi.

(a) 3 electrodes were on each subject's forehead: one was $2 \mathrm{~cm}$ above the midpoint between the eyebrows and two were above the bilateral eyebrows; (b) The two reference electrodes were mounted on the bilateral mastoid processes (right one is shown here); (c) Real-time display of the WLI and the Pi on the integrated EEG monitor, with the values updating every 2 seconds (sampling rate: $1600 \mathrm{~Hz}$ ). Pi, Pain index; WLI, wavelet index.
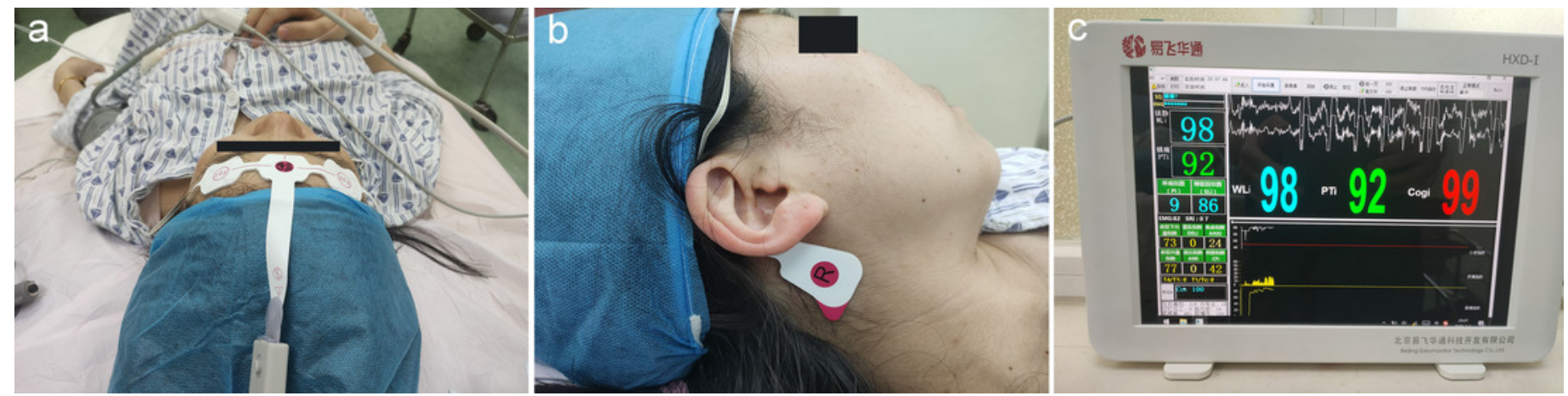
Figure 3

Correlations of Pi value with NRS score in parturients.

The expression of Pi value was positively correlated with NRS score $(r=0.768, P<0.001)$.

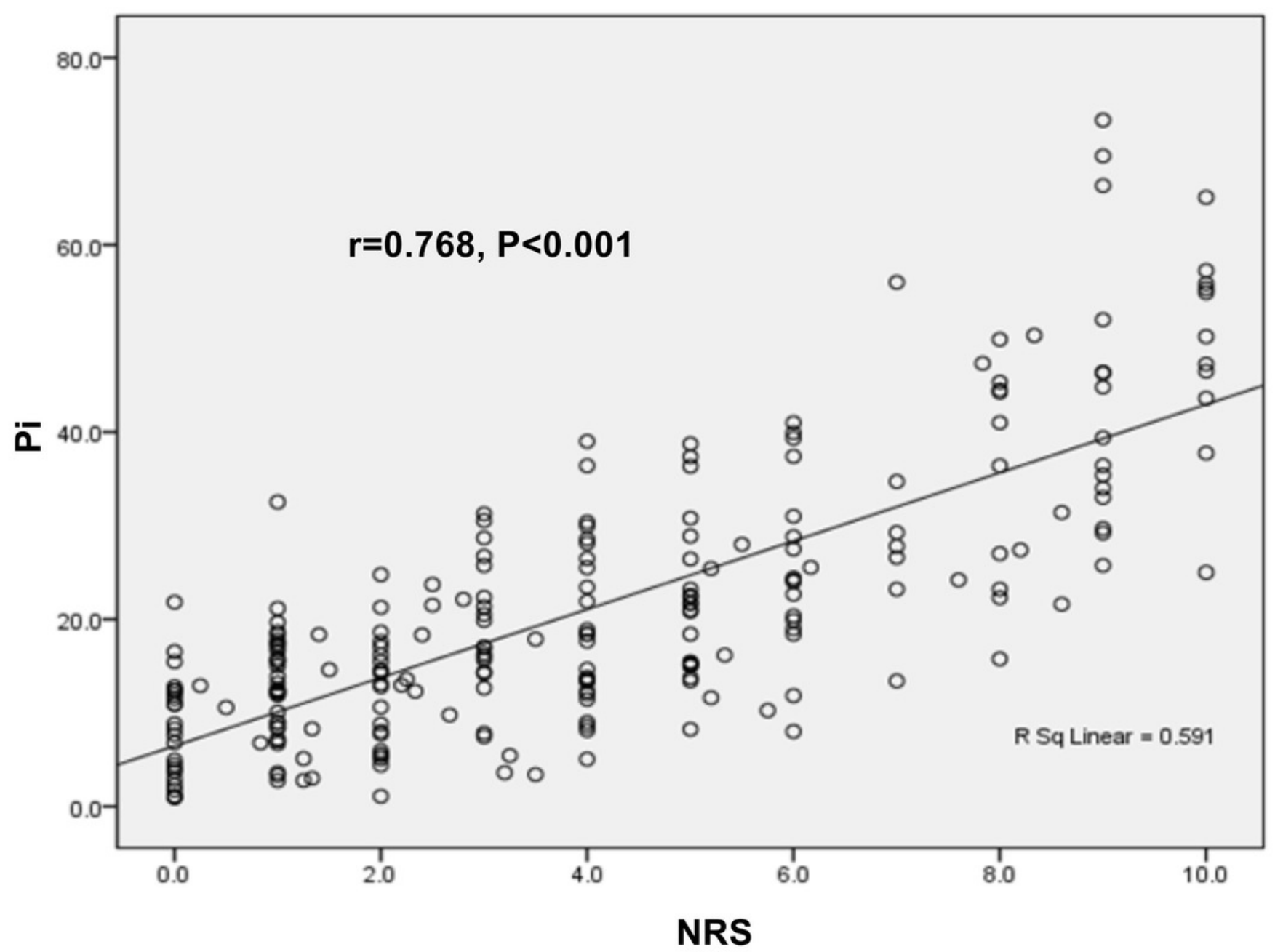




\section{Figure 4}

Subgroup analysis on correlations of Pi value with NRS score during labor based on different confounding factors.

a. the first stage of labor $(r=0.741, P<0.001)$; $b$. the second stage of labor $(r=0.774$, $P<0.001) ; c$. the presence of analgesia $(r=0.760, P<0.001)$; $d$. the absence of analgesia $(r=0.750, P<0.001)$; e. the initiation of uterine contraction $(r=0.582, P<0.001)$; $f$. the apex of uterine contraction $(r=0.751, P<0.001)$; $g$. the interval of uterine contraction $(r=0.487$, $P<0.001) ; h$. NRS score $<4(r=0.386, P<0.001) ; i$. NRS score $\geq 4$. $(r=0.688, P<0.001)$.

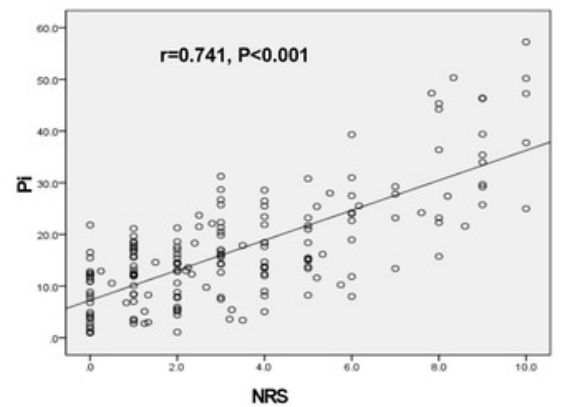

d.

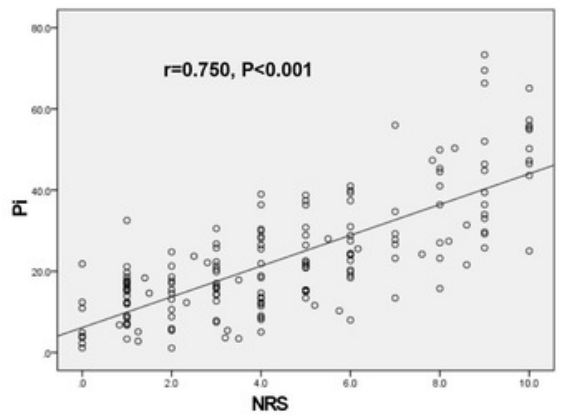

g.

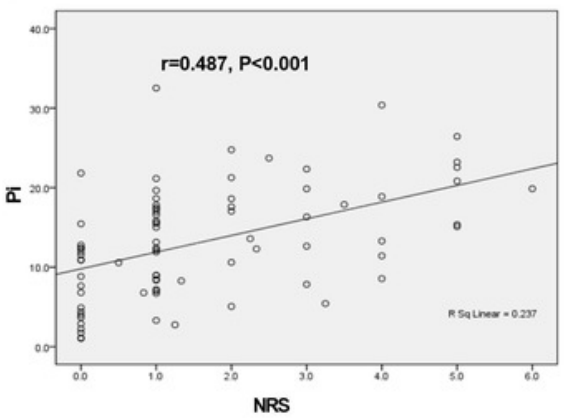

b.

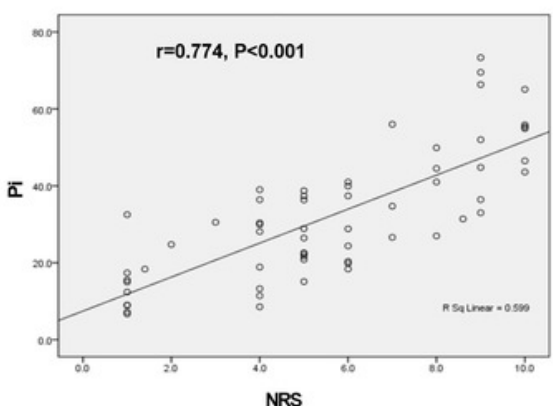

e.

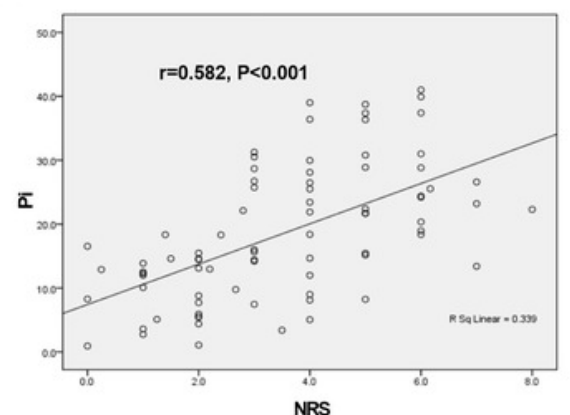

h.

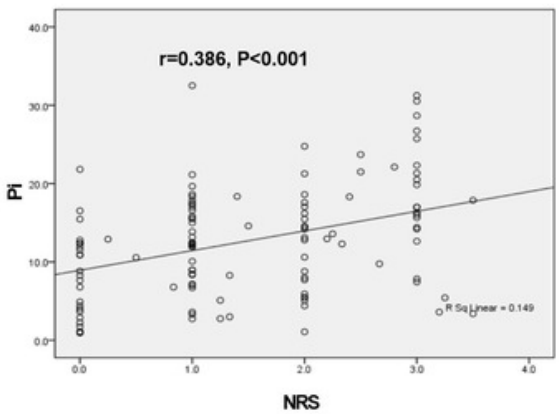

c.

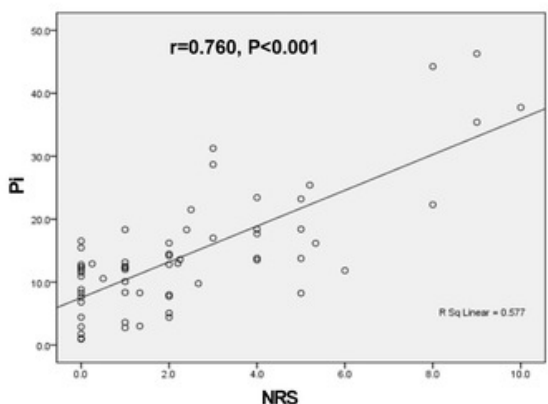

f.

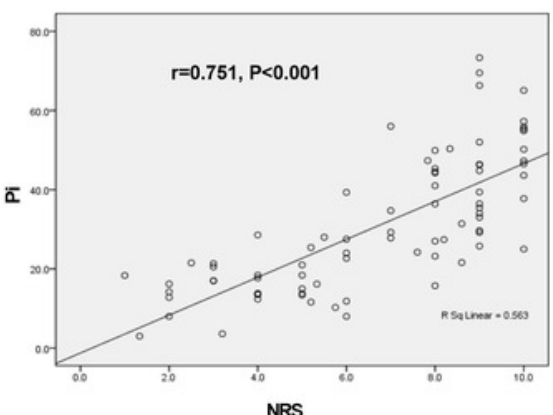

i.

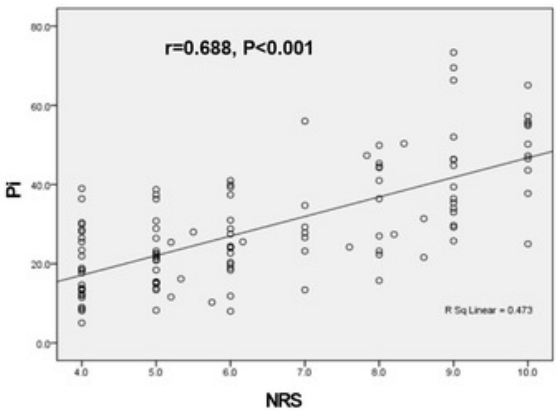




\section{Figure 5}

Overall analysis and subgroup analyses of Pi's ability to discriminate between mild and moderate-to-severe labor pain in different scenarios.

The ROC analyses were performed for the Pi in labor pain subjects, and Pi could distinguish between mild and moderate-to-severe labor pain subjects with different AUCs. a. Overall analysis containing all the parturients ( $\mathrm{AUC}=0.857,95 \% \mathrm{Cl}: 0.810-0.903$ ); $b$. the first stage of labor $(\mathrm{AUC}=0.819,95 \% \mathrm{Cl}: 0.756-0.882)$; $\mathrm{c}$. the second stage of labor $(\mathrm{AUC}=0.87,95 \% \mathrm{Cl}$ : $0.764-0.975$ ); $d$. the presence of analgesia ( $A U C=0.852,95 \% \mathrm{Cl}$ : $0.746-0.958$ ); e. the absence of analgesia ( $A \cup C=0.849,95 \% \mathrm{Cl}: 0.793-0.904$ ); $f$ the initiation of uterine contraction ( $\mathrm{AUC}=0.816,95 \% \mathrm{Cl}: 0.721-0.911) ; \mathrm{g}$. the apex of uterine contraction $(A \cup C=0.855,95 \% \mathrm{Cl}: 0.770-0.939)$; h. the interval of uterine contraction $(A \cup C=0.770,95 \%$ $\mathrm{Cl}$ : 0.634-0.906). ROC, receiver operating characteristic; AUC, area under the curve. 
a.

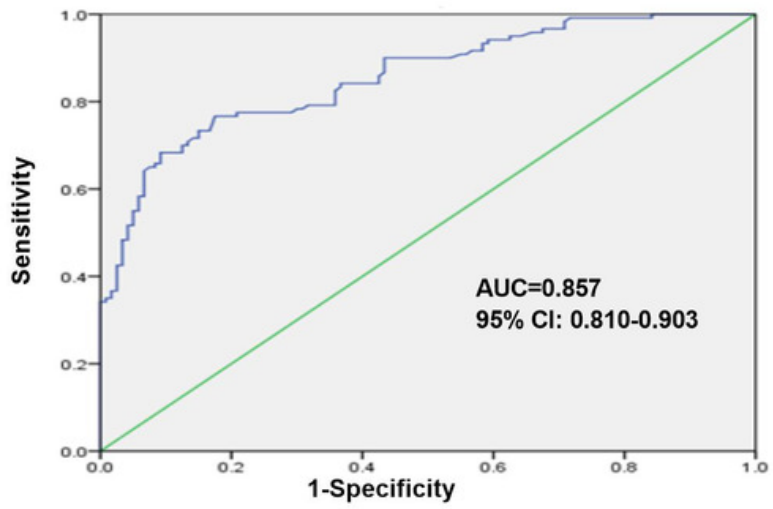

c.

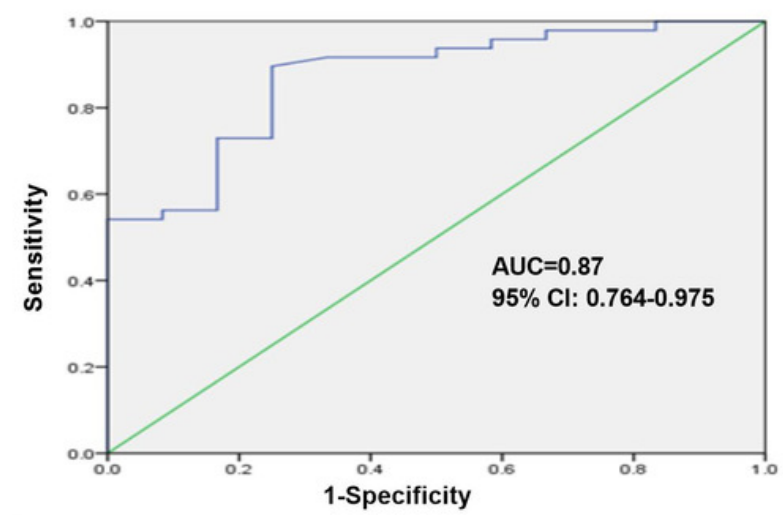

e.

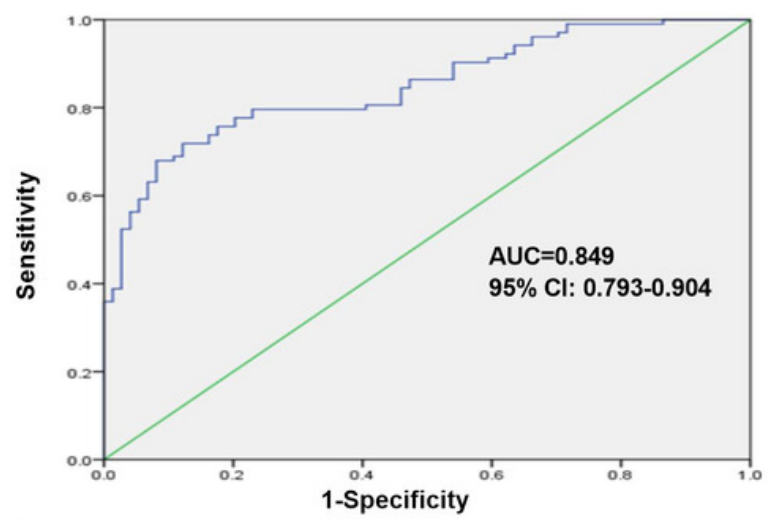

g.

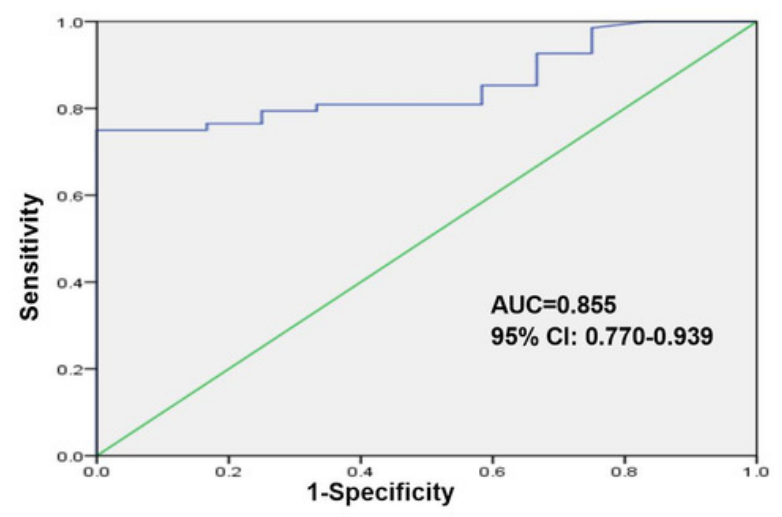

b.

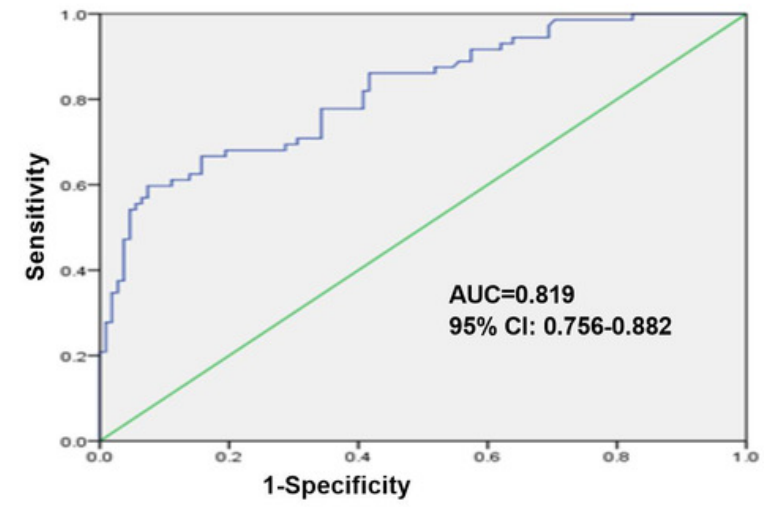

d.

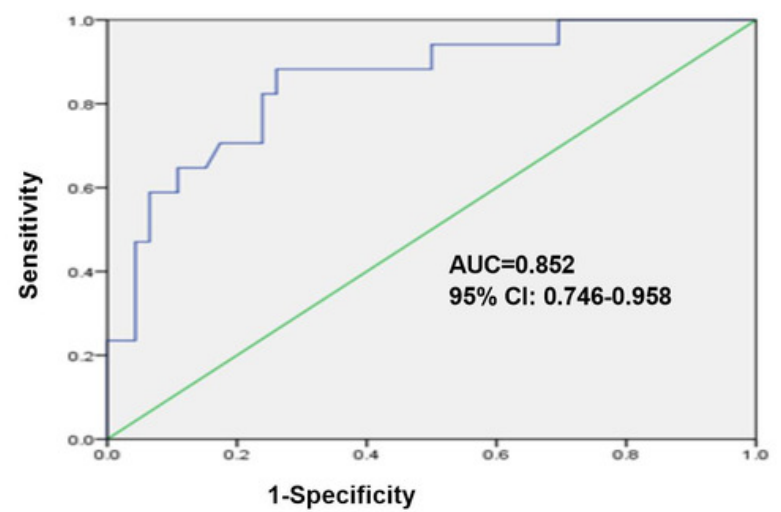

f.

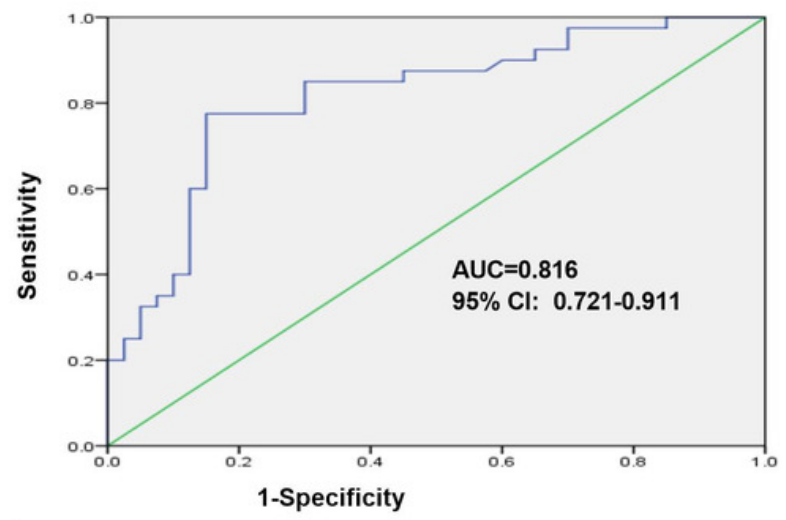

h.

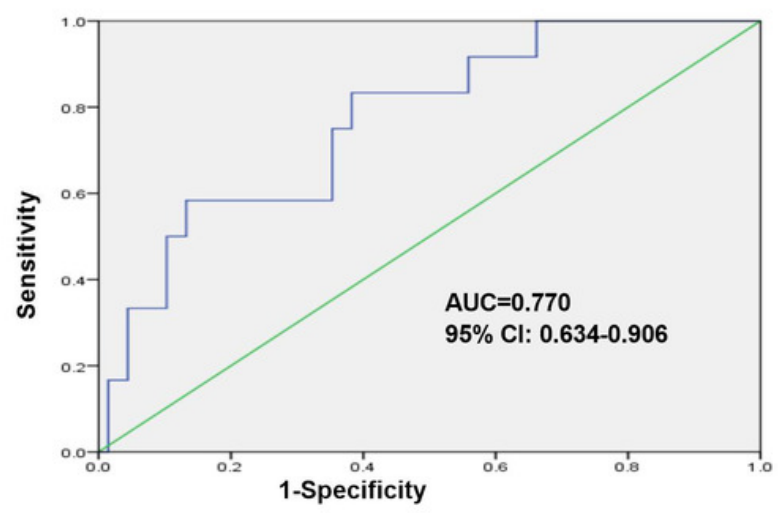

